\title{
Universal superdiffusive modes in charged two dimensional liquids
}

\author{
Egor I. Kiselev \\ Institute for Theory of Condensed Matter, Karlsruhe Institute of Technology, 76131 Karlsruhe, Germany
}

\begin{abstract}
Using a hydrodynamic approach, we show that charge diffusion in two dimensional Coulomb interacting liquids with broken momentum conservation is intrinsically anomalous. The charge relaxation is governed by an overdamped, superdiffusive plasmon mode. We demonstrate that the diffusing particles follow Lévy flight trajectories, and study the hydrodynamic collective modes under the influence of magnetic fields. The latter are shown to slow down the superdiffusive process. The results are argued to be relevant to electron liquids in solids, as well as plasmas.
\end{abstract}

\section{INTRODUCTION}

Two dimensional electron systems are among the most studied in condensed matter science: ultra-clean graphene sheets with impurity scattering lengths larger than $10 \mu \mathrm{m}$ [1] allow the observation of viscous electron flows [2] 6], which were predicted almost 50 years ago [7, 8]. Twisted bilayer graphene is on its way to become an important model system for strongly correlated electrons [9 11, and unconventional transport effects are observed in exceedingly pure delafossite metals [12 15].

Hydrodynamic transport theories have been successfully applied to predict the behavior of such systems [16[39. A particularly intriguing trait of hydrodynamics is its universality. It can be derived from general symmetry principles without knowledge of the underlying microscopic theory. This makes the hydrodynamic approach particularely interesting for the study of systems where no microscopic picture has yet been established, such as e.g. strange metals [16, 29, 38.

In this paper, the mode spectrum of a charged two dimensional liquid with weakly broken momentum conservation is investigated within a hydrodynamic framework. We find that at large scales (or equivalently small wavenumbers), the diffusion of charges is governed by a superdiffusive mode, which was described by Dyakonov and Furman [40, and which we interpret as an overdamped plasmon. This mode is shown to be universal in the sense that it does not depend on any microscopic details of the system and is determined by the rate of momentum relaxation, the charge density and the mass density alone. We also elaborate on the how the mode arises from the Lévy flight random walks of the individual charged particles. Intuitively speaking, diffusion in a charged system is faster than in an uncharged, because particles tend to spread out more due to their mutual repulsion. Using coupled Langevin equations, we show that the particle motion is dominated by Lévy flights and obeys heavy-tailed Lévy stable statistics. Furthermore, we study the influence of magentic fields on the mode spectrum and find that the superdiffusive motion is slowed down by magnetic fields.

Anomalous diffusion has been discussed in the context of Yukawa liquids and dusty plasmas. Numerical results implied that these systems are superdiffusive [41, however extensive simulations showed that the diffusion process is ultimately governed by Gaussian dynamics [42] 43. We reach a similar conclusion. In dusty plasmas, where the charged particles are screened by mobile background charges and pair interactions are well described by the Yukawa potential, ordinary diffusion prevails (see Sec. III B). However, in one component Coulomb plasmas [44] and certain colloidal suspensions [45, we expect superdiffusion as described in this paper.

\section{A. Main results}

The diffusion of charges in two dimensional systems is shown to be intrinsically anomalous due to Coulomb interactions and is described by the fractional differential equation

$$
\partial_{t} \rho_{\mathcal{Q}}=2 a \tau|\Delta|^{\frac{1}{2}} \rho_{\mathcal{Q}}
$$

which is derived in Sec. III] Here, $\rho_{\mathcal{Q}}$ is the charge density, $\tau$ is the momentum relaxation time (see Eq. (8)), $a$ is a constant depending only on the background densities of charge and mass (see Eq. (16) and below), and $|\Delta|^{\frac{1}{2}}$ is the fractional Laplacian [46, 47. Solving Eq. (1) with the initial condition $\rho_{\mathcal{Q}}(t=0, \mathbf{r})=\mathcal{Q} \delta(\mathbf{r})$, we find (Eq. 222) that the charge density follows a broadening Cauchy distribution:

$$
\rho_{\mathcal{Q}}(t, \mathbf{r})=\mathcal{Q} \frac{2 a \tau t}{2 \pi\left((2 a \tau t)^{2}+r^{2}\right)^{3 / 2}} .
$$

Eq. (2) was written down by Dyakonov and Furman in Ref. [40]. Deriving the Eqs. (1), (2) from hydrodynamics, we show that superdiffusion prevails in virtually all two dimensional, Coulomb interacting systems. Moreover, the superdiffusive behavior is universal in the sense that the only parameters that enter Eq. (1) are $a$ and $\tau$, whereas it is not influenced by the nature of the microscopic interactions. In particular, we demonstrate in Sec. IIIA that charge relaxation in quasi-relativistic Dirac systems such as pristine graphene, and twisted bilayer graphene is also superdiffusive. Here, $\tau$ has to be replaced by $\tau_{c}$ - the relaxation time of charge currents.

The Cauchy distribution of Eq. (2) is a member of the family of Lévy stable distributions. The general theory of Lévy stability (see Refs. 48, 49]) implies that if 


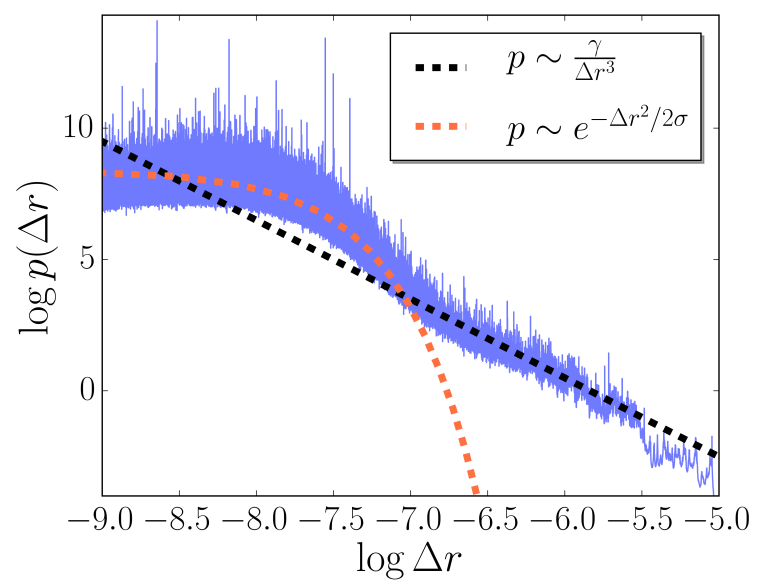

Figure 1. The Figure shows the step size distribution $p(\Delta r)$ of a random walk as performed by Coulomb interacting, diffusing particles in two dimensions. At large step sizes, the distribution clearly follows the $p \sim \Delta r^{-3}$ power-law which leads to the superdiffusive dynamics described by Eq. (1). The data was obtained by integrating the system of coupled Langevin equations of Eq. 56.

the superdiffusive dynamics of Eq. (1) emerges from the random motion of individual particles - a picture that is certainly true for classical particles - the step size distribution $p(\Delta r)$ characterizing the particles' random walks must decay as a $\Delta r^{-3}$ power-law:

$$
p(\Delta r) \sim \Delta r^{-3}, \quad \Delta r \rightarrow \infty .
$$

Such a slow power-law decay invalidates the central limit theorem, so that in the limit of many random steps, the particle distribution does not converge to a gaussian, but to the heavy-tailed Cauchy distribution of Eq. (2). Such random walks are known as Lévy flights. To demonstrate the Lévy flight nature of the charge relaxation process, we performed a computational experiment (see Sec. VI). The step size distributions of diffusing Coulomb interacting particles was studied using the coupled Langevin equations (56). As shown in Fig. 1, the numerical step size distribution indeed obeys the power-law (3), demonstrating that the particles are travelling on Lévy flight trajectories and their dynamics is governed by Eq. (1) [50, 51] at large scales. The distance travelled by the particles scales as

$$
\mathbf{r}(t) \sim 2 a \tau t
$$

which is much faster then the $\mathbf{r}(t) \sim \sqrt{2 D t}$ law of normal diffusion: the full width at half maximum of the Cauchy distribution (2) broadens with a constant velocity $v=$ $2 a \tau$.

Studying the collective mode spectrum of the charged two dimensional liquid, we show that the superdiffusive mode can be interpreted as an overdamped plasmon. In the presence of momentum relaxation, the conventional plasmon mode $\omega_{p l}=\sqrt{2 a q}$ becomes purely imaginary for small $q$ (see Fig. 22). The superdiffusive mode then emerges as an imaginary branch of the plasmon dispersion relation: $\omega_{+}=-2 i a \tau|q|$ (see Eqs. (17), (18) and (33)). In the context of electron hydrodynamics, the $\sim|q|$ dependence has been predicted for a relativistic electron hole plasma [52] (as e.g. realized in pristine graphene, see also [53]).

We also study the collective modes in the presence of magnetic fields (Sec. V), and find that superdiffusion is slowed down by a factor of $\left(1-\omega_{c}^{2} \tau^{2}\right)$, where $\omega_{c}$ is the (small) cyclotron frequency (see Eq. (55)). The relaxation of charges is then governed by the equation

$$
\partial_{t} \rho_{\mathcal{Q}}=2 a \tau\left(1-\omega_{c}^{2} \tau^{2}\right)|\Delta|^{\frac{1}{2}} \rho_{\mathcal{Q}} .
$$

Apart from the superdiffusive mode, we derive the magnetoplasmon dispersion at finite $\tau$. The magnetoplasmon dispersions are shown in Eqs. (51)-(54) and Figs. 3, 4 (see also Ref. [54]). It is noteworthy that the limits $\omega_{c} \rightarrow 0$ and $q \rightarrow 0$ are not interchangeable and result in different dispersion relations. This behavior is discussed below Eq. 48.

In Sec. IV, we discuss the connection between the Einstein relation and the superdiffusive behavior. We derive the Einstein relation

$$
D=\frac{\sigma_{\|}(\omega \rightarrow 0,0)}{\chi_{\rho_{\mathcal{Q}} \rho_{\mathcal{Q}}}(0, \mathbf{q} \rightarrow 0)},
$$

where $\chi_{\rho_{\mathcal{Q}} \rho_{\mathcal{Q}}}$ is the charge susceptibility, from hydrodynamics, showing that the diffusion constant $D$ appears in a diffusive pole of the nonlocal longitudinal conductivity $\sigma_{\|}$. Despite the presence of the diffusive pole $\omega_{D}=-i D q^{2}$, the equilibration of inhomogeneous charge or current distributions is superdiffusive, and is not described by an ordinary diffusion equation. This changes when a gate is located in the vicinity of the two dimensional system. If the distance between the gate and the $2 \mathrm{D}$ system is sufficiently small, the long range Coulomb potential becomes subleading to the capacitance of the gate. In this case, the relaxation of charges indeed follows a diffusion equation, and the Einstein relation gives the diffusion constant (see Sec. IV C).

Finally, the contribution of the superdiffusive mode the specific heat of a two dimensional liquid was calculated (Sec. VII). At low temperatures we find

$$
c_{V}=c_{1} T-c_{2} T^{2}+c_{3} T^{3}-\mathcal{O}\left(T^{5}\right),
$$

where all higher order terms are of odd powers in $T$. The coefficients are given by $c_{1}=\frac{q^{*}}{24 a \tau}, c_{2}=\frac{3 \zeta(3)}{8 \pi a^{2} \tau^{2}}$, where $q^{*}$ is a momentum cut-off. The coefficent in front of the $T^{2}$ term does not depend on $q^{*}$. This term is due to the non-analyticity of the superdiffusive mode at $q=0$ (see Eq. (60)). The result (5) is very different from the $\sim T^{4}$ specific heat of the undamped plasmon mode $\omega_{p l}=\sqrt{2 a q}$ [55]. For a normal diffusive mode in two dimension we find

$$
c_{V, g}=d_{1} T+d_{1}^{\prime} T \log \left(\frac{1}{T}\right)+d_{3} T^{3}+\mathcal{O}\left(T^{5}\right) .
$$


The $T \log (1 / T)$ contribution, which is dominant at low temperatures is not uncommon for two dimensional systems. In $\mathrm{Sr}_{3} \mathrm{Ru}_{2} \mathrm{O}_{7}$, the $T \log (1 / T)$ contribution has been observed experimentally [56]. Other mechanisms leading to a $T \log (1 / T)$ dependence of the specific heat are quantum critical fluctuations of overdamped bosonic modes with a dynamical exponent $z=2$ [57] and scattering between hot Fermi pocket and cold Fermi surface electrons in $\mathrm{Sr}_{3} \mathrm{Ru}_{2} \mathrm{O}_{7}$ 58.

In experiments, measurements of the superdiffusive modes could involve pump probe setups which can monitor the relaxation of charge carriers at very short timescales (see e.g. [59]). If surplus charge is induced at a given point $\mathbf{r}_{0}$, this charge will relax as described by $(2)$. The charge density right at $\mathbf{r}_{0}$ will decay accorting to $\rho\left(\mathbf{r}_{0}, t\right) \sim 1 / t^{2}$. For Gaussian diffusion, the decay at $\mathbf{r}_{0}$ scales as $\sim 1 / t$, which is much slower for small $t$. The optical properties of the material, measured by the probe signals, will follow this dynamics. Time of flight measurements can be another way to probe the superdiffusive behavior. Such measurements are used to measure electron drift velocities in the presence of homogeneous electric fields 60, 61 and could be used to probe the $r \sim t$ scaling of Eq. (4) (vs. $r \sim \sqrt{t}$ in the Gaussian case) directly.

The remainder of this paper is organized as follows: in Sec. II we introduce the hydrodynamic framework that is used throughout the paper. Sec. III presents a derivation of the Eqs. (1) and (2). Yukawa liquids and two dimensional Dirac systems are discussed. Sec. IV] deals with the Einstein relation and with gated $2 \mathrm{D}$ systems. The influence of magnetic fields on the spectrum of collective modes is investigated in Sec. V. Sec. VI presents the numerical results on the Langevin dynamics of charged particles. Finally, Sec. VII deals with the contributions of collectives modes to the specific heat.

\section{HYDRODYNAMICS}

The motion of a charged two dimensional liquid are governed by the laws of momentum and charge conservation and the corresponding continuity equations. Introducing the flow velocity $\mathbf{u}$, the charge denstiy $\rho_{\mathcal{Q}}$ and mass density $\rho_{\mathcal{M}}$, we can write the charge current as $j_{\mathcal{Q}, i}=\rho_{\mathcal{Q}} u_{i}$, and the momentum density as $g_{i}=\rho_{\mathcal{M}} u_{i}$. In the case of a Galilean invariant system, we have $\rho_{\mathcal{M}}=m \rho, \rho_{\mathcal{Q}}=e \rho$, where $\rho$ is the particle number density and $m, e$ are the mass and charge of the particles that constitute the liquid. If the Galilean invariance is broken, $\mathbf{u}$ can be introduced as a field sourcing the conserved crystal momentum, and the densities $\rho_{\mathcal{Q}}$ and $\rho_{\mathcal{M}}$ can be defined using the memory matrix formalism [21, 38, (see Appendix A for details).

The hydrodynamic equations that we will use in the following are continuity equation for the charge density $\rho_{\mathcal{Q}}:$

$$
\partial_{t} \rho_{\mathcal{Q}}+\partial_{i}\left(\rho_{\mathcal{Q}} u_{i}\right)=0
$$

and the Navier-Stokes equation, which is the continuity equation for the momentum density:

$$
\partial_{t}\left(\rho_{\mathcal{M}} u_{i}\right)+\partial_{j} \Pi_{i j}=-\frac{1}{\tau} \rho_{\mathcal{M}} u_{i}-\rho_{\mathcal{Q}} \nabla \phi
$$

Here, $\Pi_{i j}$ is the momentum current tensor and $\phi$ is the electrostatic potential. $\tau$ is the relaxation time of the momentum density and accounts for momentum dissipation, e.g. due to impurities. The above equations are very similar to the equations of classical hydrodynamics [62, 63. However, since charged liquids are considered, we need to take care of electrostatic forces induced by an inhomogeneous charge density. The electrostatic potential $\phi$ in Eq. (8) therefore depends not only on externally applied fields but also on the charge density: $\phi=\phi\left[\rho_{\mathcal{Q}}\right]$. In general one can write

$$
\Pi_{i j}=\rho_{\mathcal{M}} u_{j} u_{i}+p \delta_{i j}-\tau_{i j}
$$

Here, $\rho_{\mathcal{M}}$ is the mass density, $p$ is the fluid's pressure. The viscous stress tensor $\tau_{i j}$ can be written as $\tau_{i j}=$ $\eta_{i j k l} \partial_{k} u_{l}$ using the viscosity tensor $\eta_{i j k l}$. We limit ourself to isotropic systems where the viscosous stress tensor can be written in terms of the shear viscosity $\eta$ and bulk viscosity $\zeta$ :

$$
\tau_{i j}=\eta \partial_{j} \partial_{j} u_{i}+\zeta \partial_{i} \partial_{j} u_{j}
$$

The hydrodynamic equations (7), (8) describe the macroscopic dynamics of translation invariant fluids with or without Galilean invariance without making assumptions on the nature of microscopic interactions. Therefore, they are useful tools to study the dynamics of strange metals and other materials where a microscopic theory is currently out of reach [16, 19, 38].

\section{SUPERDIFFUSION}

Particles undergoing normal diffusion spread in space according to the law $r \sim t^{1 / 2}$. Superdiffusion, on the other hand, is characterized by a faster dynamics: $r \sim$ $t^{1 / \alpha}$, where $0<\alpha<2$. If we picture diffusion as a random walk of colliding particles, its speed crucially depends on the so called step size distribution: the distribution of distances that particles travel between collisions. If this distribution has a finite variance, the resulting diffusion process will always be Gaussian for large times and will obey the $r \sim t^{1 / 2}$ scaling, which is a consequence of the central limit theorem. If, on the other hand, the step sizes are distributed according to a power law and their variance is infinite, we enter the realm of superdiffusion. Another important notion is that of stability. A distribution function $p(r)$ is called stable (sometimes Lévy-stable or $\alpha$-stable) if the sum of random variables $r=\left(1 / c_{n}\right) \sum_{i}^{n} r_{i}$, with each $r_{i}$ distributed according to $p(r)$, is itself distributed according to $p(r)$. It can be shown that $c_{N}=n^{1 / \alpha}$ is the only possible choice [49]. 
Letting $n=t / \Delta t$, where $\Delta t$ is the time between collisions, we obtain the $r \sim t^{1 / \alpha}$ scaling mentioned above, where $\alpha=2$ again corresponds to a Gaussian distribution. In this sense superdiffusion is a generalization of normal diffusion for heavy-tailed step size distributions with infinite variance [48, 49. In this section, we describe how superdiffusive modes arise in two dimensional materials from the hydrodynamic equations introduced in the previous section.

In the following it will be useful to separate the densities $\rho_{\mathcal{Q}}, \rho_{\mathcal{M}}$ into a homogeneous background and a small fluctuating term:

$$
\rho_{\mathcal{Q} / \mathcal{M}}=\rho_{\mathcal{Q} / \mathcal{M}}^{(0)}+\rho_{\mathcal{Q} / \mathcal{M}}^{(1)}(t, \mathbf{r})
$$

In the absence of external fields, the electrostatic potential is determined by the inhomogeneous part of the charge density:

$$
\phi(\mathbf{r})=\frac{1}{\varepsilon} \int d^{2} x^{\prime} \frac{\rho_{\mathcal{Q}}^{(1)}(t, \mathbf{r})}{\left|\mathbf{r}-\mathbf{r}^{\prime}\right|}
$$

where $\varepsilon$ is the dielectric constant of the substrate. The potential $\phi$ in Eqs. (8) and 12 is the hydrodynamic analogue of the self-consistent potentials of the Landau-Silin 64 and Vlasov 65] theories. After a Fourier transform the above equation reads

$$
\phi(\mathbf{q})=\frac{1}{\varepsilon} V(\mathbf{q}) \rho_{\mathcal{Q}}^{(1)}(\omega, \mathbf{q})
$$

with $V(\mathbf{q})=2 \pi / q$. We will be interested in the system's response to small inhomogeneities at small q. Let us therefore sort out the higher order terms. The pressure term in Eq. 80 can be written as $\nabla p=\left(K / \rho_{\mathcal{Q}}^{(0)}\right) \nabla \rho_{\mathcal{Q}}^{(1)}$ with the bulk modulus $K=\rho_{\mathcal{Q}}\left(\partial p / \partial \rho_{\mathcal{Q}}\right)$. Using the continuity equation for $\rho_{\mathcal{Q}}$, we find that the pressure term is of order $q^{2}: \nabla_{i} p \propto q_{i} q_{j} u_{j}$. The viscous terms $\partial_{j} \tau_{i j}$ also are of order $q^{2}$. On the other hand, using Eq. 7) one finds

$$
\rho_{\mathcal{Q}}^{(1)}(\omega, \mathbf{q})=\frac{q_{i}}{\omega} \rho_{\mathcal{Q}}^{(0)} u_{i}(\omega, \mathbf{q})
$$

Therefore, linearizing Eq. 8 in $u_{i}$ and $\rho_{\mathcal{Q} / \mathcal{M}}^{(1)}$ and performing a Fourier transform we obtain, to first order in $\mathbf{q}$

$$
\left(-i \omega+\tau^{-1}\right) u_{i}=-\frac{\left(\rho_{\mathcal{Q}}^{(0)}\right)^{2}}{\rho_{\mathcal{M}}^{(0)} \varepsilon}\left(i q_{i}\right) V(\mathbf{q}) \frac{q_{j}}{\omega} u_{j} .
$$

Being interested in the longitudinal solutions to Eq. (14, we set $\mathbf{u} \propto \mathbf{q}$. The above equation then reduces to

$$
\omega\left(i \omega-\tau^{-1}\right)=\frac{i\left(\rho_{\mathcal{Q}}^{(0)}\right)^{2}}{\rho_{\mathcal{M}}^{(0)} \varepsilon} q^{2} V(\mathbf{q}) .
$$

It follows

$$
\omega_{ \pm}=-\frac{i}{2 \tau} \pm \sqrt{2 a q-\frac{1}{4 \tau^{2}}}
$$

with $a=\pi\left(\rho_{\mathcal{Q}}^{(0)}\right)^{2} /\left(\varepsilon \rho_{\mathcal{M}}^{(0)}\right)$. In the absence of momentum dissipation, i.e. in the limit $\tau \rightarrow \infty$, Eq. 16 reduces to the well known $2 \mathrm{D}$ plasmon dispersion $\omega=\sqrt{2 a q}$ [66. Eq. 16 describes a damped out plasmon mode, which is purely imaginary below a threshold wavevector $q^{*}=1 /\left(8 \tau^{2} a\right)$. This purely imaginary branch of the dispersion corresponds to a superdiffusive mode, as we will shortly see. For Fermi liquids, Eq. [15), which describes the plasmon pole in the presence of disorder, has been derived diagrammatically in Ref. 67]. As shown here, it can be justified on much more general grounds. Expanding Eq. 16 for small $q$ we find

$$
\begin{aligned}
& \omega_{-} \approx-\frac{i}{\tau}+2 i a \tau|q| \\
& \omega_{+} \approx-2 i a \tau|q| .
\end{aligned}
$$

Finally, there exists a transverse mode with $\mathbf{u} \cdot \mathbf{q}=0$ which is given by

$$
\omega_{\perp}=-i / \tau+\mathcal{O}\left(q^{2}\right) .
$$

The dispersion relation of Eq. 18 describes a superdiffusive mode for the charge density $\rho_{\mathcal{Q}}$. In contrast to the $|q|$ dependence of Eq. [18, , simple diffusive modes are governed by a dispersion relation $\omega=-i D q^{2}$, where $D$ is the diffusion constant. In space time coordinates this translates to the well known diffusion equation $\partial_{t} \rho_{\mathcal{Q}}=D \nabla^{2} \rho_{\mathcal{Q}}$. On the other hand, Eq. 18 , via Eq. (14), leads to a fractional diffusion equation for the charge density:

$$
\partial_{t} \rho_{\mathcal{Q}}=2 a \tau|\Delta|^{\frac{1}{2}} \rho_{\mathcal{Q}}
$$

The fractional laplace operator $|\Delta|^{\frac{\alpha}{2}}$ is defined via it's properties under the Fourier transform: $\mathcal{F}\left[|\Delta|^{\frac{\alpha}{2}} f\right](q)=$ $-|q|^{\alpha} \mathcal{F}[f](q)$ 46, 47]. The special case $\alpha=1$ is used in Eq. 20.

Fractional diffusion equations [51] are used to describe superdiffusion in systems as different as random media [68] and financial markets [69, and can be motivated by general symmetry considerations 70 . The fractional diffusion equation that we arrived at can be interpreted as the continuous time limit of a stochastic process involving Lévy flights. To see this, let us solve Eq. 20. Taking the Fourier transform of the spatial portion of the equation and using the initial condition $\rho_{\mathcal{Q}}\left(t_{0}, \mathbf{r}\right)=\mathcal{Q} \delta(\mathbf{r})$ where $\mathcal{Q}$ is the charge, we find

$$
\rho_{\mathcal{Q}}(\Delta t, \mathbf{q})=\mathcal{Q} e^{-2 i a \tau|q| \Delta t}
$$

Here, we have abbreviated $\Delta t=t-t_{0}$. Taking the inverse Fourier transform one obtains

$$
\rho_{\mathcal{Q}}(\Delta t, \mathbf{r})=\mathcal{Q} \frac{2 a \tau \Delta t}{2 \pi\left((2 a \tau \Delta t)^{2}+r^{2}\right)^{3 / 2}} .
$$


This function is interpreted as the probability distribution for the distances a particle travels in a period of time $\Delta t$ starting at $\mathbf{r}=0$, i.e. the step size distribution of a random walk. Its mean value vanishes by symmetry and its variance is infinite: $\left\langle r^{2}\right\rangle=\infty$, while its width grows linearly with $t$. The distance $r$ that a particle travels therefore scales as

$$
r \sim t,
$$

which is much faster than for a normal diffusion processes, where the distance scales as $r \sim t^{1 / 2}$.

In general, Lévy flights in $d$ dimensions are characterized by heavy-tailed power-law step size distributions which scale as 71

$$
p(r) \sim r^{-(\alpha+d)}
$$

for large step sizes $r$ [48, 49]. The word "flight" is used to stress that due to its slow decay for $r \rightarrow \infty, p(r)$ allows for very large steps which would be extremely improbable for normally distributed step sizes. The exponent $\alpha$ with $0<\alpha<2$ fully characterizes the Lévy stable distribution function [49] and the distance travelled by a random walker scales according to $r \sim t^{\alpha}$, as described in the beginning of this section. Thus Eqs. (18) and 20 indeed describe a Lévy flight with exponent $\alpha=1$. Another way to see that Eq. (20) describes a Lévy flight is to remember that the characteristic function of a (symmetric) Lévy stable distribution is

$$
\left\langle e^{-i \mathbf{q} \cdot \mathbf{r}}\right\rangle=e^{-\gamma|q|^{\alpha}}
$$

where $\gamma$ characterizes the width of the distribution 48 , 49. For $\alpha=1$ this indeed corresponds to the solution of Eq. 20) in Fourier space given in Eq. (21).

\section{A. Dirac liquids}

The prime example of a Dirac liquid is graphene at the charge neutrality point. At finite temperatures, equal numbers of particles and holes are excited, such that the system remains charge neutral. Thus $\rho_{\mathcal{Q}}^{(0)}=0$ holds. Homogeneous electric currents consist of equal numbers of electrons and holes. However at finite wavevectors, $\rho_{\mathcal{O}}^{(1)}=\frac{q_{i}}{\omega} j_{\mathcal{Q}, i}$ holds, such that the self-consistent potential 12 must be included [72. In charge neutral graphene, electric currents are relaxed by interaction effects since they are not protected by momentum conservation [73]. The corresponding relaxation time $\tau_{c}$ damps out the plasmon mode just as $\tau$ does in Eq. (16). The collective mode structure of this system was studied in Ref. [72]. The damped plasmon mode is given by

$$
\omega_{ \pm}=-\frac{i}{2 \tau_{c}} \pm \sqrt{\frac{v q}{\tau_{V}}-\frac{1}{4 \tau_{c}^{2}}} .
$$

Here, $v$ is the electron group velocity and $\tau_{V}=\frac{2 \pi k_{B} T \hbar}{\alpha N \log (2)}$ with the fine structure constant $\alpha=\frac{e^{2}}{\varepsilon v \hbar} \cdot \tau_{V}$ characterizes the strength of the electrostatic repulsion. For small $q$ we find a superdiffusive mode

$$
\omega_{+}=-i \frac{v \tau_{c}}{\tau_{V}} q .
$$

Similar physics will prevail in other charge neutral systems such as twisted bilayer graphene (TBG), since at small wavenumbers the electric current will always follow the dynamics $\left(\partial_{t}+\tau\right) j_{\mathcal{Q}}^{(1)}(t, \mathbf{r})=-\nabla \int d^{2} x^{\prime} \frac{\rho_{\mathcal{Q}}^{(1)}\left(t, \mathbf{r}^{\prime}\right)}{\varepsilon\left|\mathbf{r}-\mathbf{r}^{\prime}\right|}$, which, together with the continuity equation will result in a superdiffusive mode. The conclusion that charge relaxation in pristine graphene is a Cauchy process was first reached by Kolomeisky and Straley in Ref. [53] extending the original arguments of Ref. [40]. Interestingly, the phase space behavior of Dirac liquids is also superdiffusive [74].

An estimation of the value $q^{*}=\tau_{V} /\left(4 \tau_{c}^{2} v\right)$ below which superdiffusion prevails in charge neutral graphene can be made with the scattering times calculated in Ref. [72]: $\tau_{V}=2 \pi^{2} \hbar /\left(4 \alpha k_{B} T \ln (2)\right), \tau_{c}=$ $\ln (2) \hbar /\left(0.8 \cdot \alpha^{2} k_{B} T\right)$ and $v \approx 10^{6} \mathrm{~m} / \mathrm{s}$. Here $\alpha$ is the fine structure constant. We obtain $q^{*} \approx 3 \cdot 10^{5} \frac{1}{\mathrm{~m}}$, where we used a temperature of $T=50 \mathrm{~K}$ and a substrate dielectric constant of $\varepsilon=6$, gaving $\alpha \approx 0.1$. The above value of $q^{*}$ corresponds to sample lengths of tens of micrometers. Notice however, that $q^{*} \sim \alpha^{3}$. Thus the typical lengthscales of the superdiffusive regime will strongly decrease for larger values of $\alpha$, as they are typical for TBG.

It should be noted, that interlayer coupling in Moiré systems can lead to a non-trivial dependence of the dielectric function on $q$, leading to unconventional screened interactions [75, 76]. Such effects are not captured by our hydrodynamic theory, but could be an interesting topic for further study.

\section{B. Yukawa liquids}

In a Yukawa liquid charges interact with a Yukawa pair-potential

$$
V_{Y}(\mathbf{q})=\frac{2 \pi}{q+\kappa},
$$

where $\kappa$ is the inverse screening length. Such a screened interaction potential arises, when the considered charges are screened by mobile background charges, as for example in dusty plasmas [77]. Two dimensional Yukawa liquids are widely studied (see e.g. [44, 78, 79]). In particular, superdiffusion has been discussed [41, but ultimately ruled out in favor of normal diffusion [42.

Within our hydrodynamic model, it is readily shown that diffusion in a 2D Yukawa liquid is indeed Gaussian. To this end we replace $V(\mathbf{q})$ in Eq. 15 by the Yukawa 
potential 27. Instead of Eq. 16 we then obtain

$$
\begin{aligned}
& \omega_{+}^{Y}=-\frac{i}{2 \tau}+\sqrt{\frac{2 a q^{2}}{q+\kappa}-\frac{1}{4 \tau^{2}}} . \\
& \omega_{-}^{Y}=-\frac{i}{2 \tau}-\sqrt{\frac{2 a q^{2}}{q+\kappa}-\frac{1}{4 \tau^{2}}}
\end{aligned}
$$

For small $q$, Eq. 28 reduces to

$$
\begin{aligned}
& \omega_{-}^{Y} \approx-\frac{i}{\tau}+i \frac{2 a \tau}{\kappa} q^{2} \\
& \omega_{+}^{Y} \approx-i \frac{2 a \tau}{\kappa} q^{2} .
\end{aligned}
$$

The mode $\omega_{+}$describes normal diffusion where the diffusion constant is given by $D_{Y}=\frac{2 a \tau}{\kappa}$. If $\kappa$ is large, the bulk and shear viscosities, which also give a contribution of order $\mathcal{O}\left(q^{2}\right)$ will enter the expression for the diffusion constant. Eq. (29) is therefore a good approximation, if the screening is weak, i.e. $\kappa \rightarrow 0$. In this case, the dynamics of a weakly inhomogeneous charge distribution is described by the diffusion equation

$$
\partial_{t} \rho_{\mathcal{Q}}=D \nabla^{2} \rho_{\mathcal{Q}}
$$

\section{BEHAVIOR AT LARGER WAVENUMBERS, EINSTEIN RELATION, GATING}

\section{A. Behavior at $\mathcal{O}\left(q^{2}\right)$}

The full dispersion relations $\omega_{ \pm}(q)$ which are also valid at larger $q$ can be efficiently obtained from the well known condition for collective excitations $\varepsilon(\omega, \mathbf{q})=0$, where $\varepsilon(\omega, \mathbf{q})=\phi_{\operatorname{ext}}(\omega, \mathbf{q}) / \phi(\omega, \mathbf{q})$ is the dielectric function of the $2 \mathrm{D}$ material. Here $\phi=\phi_{\text {ext }}+\phi_{\text {ind }}$ is the total electric potential, where $\phi_{\text {ext }}$ is due to external sources and $\phi_{\text {ind }}$ is sourced by the inhomogenious charge carrier density $\rho_{\mathcal{Q}}^{(1)}$. From the definition of $\varepsilon$ we find

$$
\varepsilon(\omega, \mathbf{q})=1-\chi_{\rho_{\mathcal{Q}} \rho_{\mathcal{Q}}}(\omega, \mathbf{q}) V(\mathbf{q}) .
$$

Here $\chi_{\rho_{\mathcal{Q}} \rho_{\mathcal{Q}}}$ is the charge susceptibility which is defined via the relation

$$
\rho_{\mathcal{Q}}^{(1)}(\omega, \mathbf{q})=\chi_{\rho_{\mathcal{Q}} \rho_{\mathcal{Q}}}(\omega, \mathbf{q}) \phi(\omega, \mathbf{q}) .
$$

The condition $\varepsilon(\omega, \mathbf{q})=0$ together with the Eqs. (35) and (37) then gives

$$
\omega_{ \pm}(q)=-i \frac{1}{2 \tau}-i q^{2} \frac{\zeta+\eta}{2 \rho_{\mathcal{M}}^{(0)} \tau} \pm \sqrt{2 a q\left(1+\frac{K q}{2 a \rho_{\mathcal{M}}^{(0)}}\right)-\frac{1}{4 \tau^{2}}\left(\frac{q^{2}(\zeta+\eta)}{\rho_{\mathcal{M}}^{(0)}}+1\right)^{2}}
$$

For small $q$, Eq. 33 reduces to the expression given in Eq. (16). A third mode $\omega_{\perp}$ is easily found by setting $\mathbf{u}$. $\mathbf{q}=0$ in the Navier-Stokes equation (8). This transverse mode obeys the dispersion relation

$$
\omega_{\perp}=-\frac{i}{\tau}-i \eta q^{2} .
$$

The modes of Eqs. (33), 34) are shown in Fig. 2

\section{B. Einstein relation}

Although the charge dynamics in a two dimensional liquid is superdiffusive, transport coefficients obey the Einstein relation: The longitudinal conductivity $\sigma_{\|}$is defined via Ohm's law: $j_{\mathcal{Q}, i}(\omega, \mathbf{q})=\sigma_{\|}(\omega, \mathbf{q}) E_{i}(\omega, \mathbf{q})$ with $\mathbf{E} \propto \mathbf{q}$, where the electric field is determined by the gradient of the total electrostatic potential: $\mathbf{E}=-\nabla \phi$. In the regime of linear response, we find from Eq. (8)

$$
\sigma_{\|}(\omega, \mathbf{q})=\frac{\tau\left(\rho_{\mathcal{Q}}^{(0)}\right)^{2} / \rho_{\mathcal{M}}^{(0)}}{1-i \omega \tau+i \tau \frac{q^{2}}{\omega} \frac{K}{\rho_{\mathcal{M}}^{(0)}}+q^{2} \frac{\eta+\zeta}{\rho_{\mathcal{M}}^{(0)}}} .
$$

Here we have again used the relation $\nabla p=$ $\left(K / \rho_{\mathcal{Q}}^{(0)}\right) \nabla \rho_{\mathcal{Q}}^{(1)}$ where $K=\rho_{\mathcal{Q}}\left(\partial p / \partial \rho_{\mathcal{Q}}\right)$ is the bulk modulus. We also assumed an isotropic system where the viscous stress tensor reduces to $\tau_{i j}=\eta \partial_{j} \partial_{j} u_{i}+\zeta \partial_{i} \partial_{j} u_{j}$, with the shear and bulk viscosities $\eta, \zeta$. Let us relate the bulk modulus to the charge susceptibility $\chi_{\rho_{\mathcal{Q}} \rho_{\mathcal{Q}}}$. In the static case $\omega=0$, forces stemming from fluctuations of $\phi(\mathbf{x})$ are balanced by pressure changes: $-i \mathbf{q} \rho_{\mathcal{Q}}^{(0)} \phi(0, \mathbf{q})=$ $\left(K / \rho_{\mathcal{Q}}^{(0)}\right) i \mathbf{q} \rho_{\mathcal{Q}}^{(1)}(0, \mathbf{q})$. It follows

$$
\chi_{\rho_{\mathcal{Q}} \rho_{\mathcal{Q}}}(0, \mathbf{q} \rightarrow 0)=-\left(\rho_{\mathcal{Q}}^{(0)}\right)^{2} K^{-1} .
$$

This is a special case of the relation

$$
\sigma_{\|}(\omega, \mathbf{q})=\frac{i \omega}{q^{2}} \chi_{\rho_{\mathcal{Q}} \rho_{\mathcal{Q}}}(\omega, \mathbf{q}),
$$

which can be obtained from the Kubo expression for $\sigma$ and the continuity equation [80. The conductivity (35) has a diffusive pole

$$
\omega_{D}(q)=-i D q^{2}
$$

for small $q$ (see Eq. 40 for the full expression). Here, $D$ is the diffusion constant $D=K \tau / \rho_{\mathcal{M}}^{(0)}$. However, the pole (38) does not coincide with the superdiffusive mode $\omega_{+}(q)$ of Eq. 18), because the electric conductivity characterizes the system's response to the total electric field 

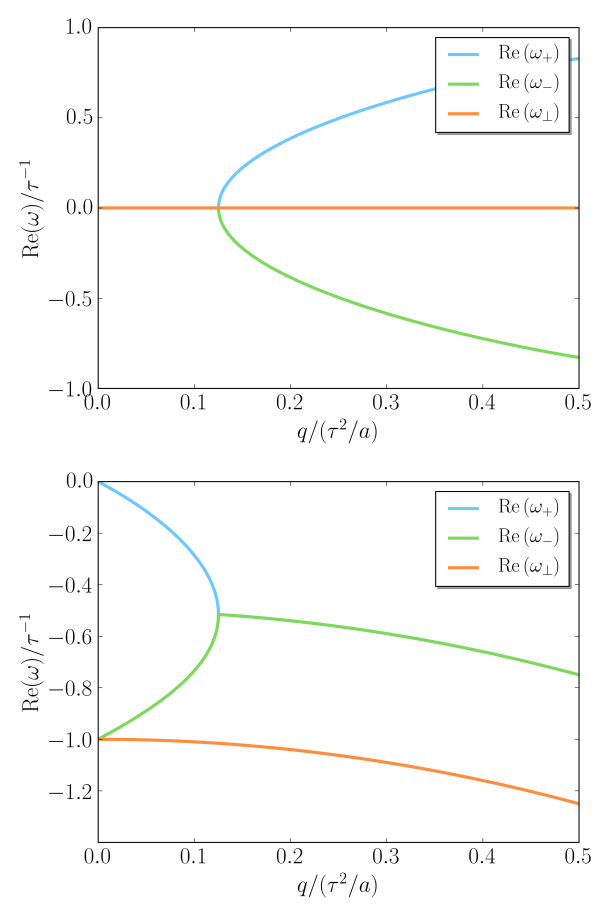

Figure 2. The dispersion relation of the damped plasmon mode is shown. In the presence of momentum relaxation, the well known $\sqrt{q}$-plasmon mode is damped out, such that below a certain threshold wavenumber $\operatorname{Re}\left(\omega_{ \pm}(q)\right)=0$ holds. Interestingly, the plasmon is also damped out at large $q$, due to both viscosity and momentum relaxation. The blue line corresponds to the superdiffusive mode $\omega_{+}(q)$ (see Eq. [18)).

$\mathbf{E}=-\nabla \phi$. For a given electrostatic potential $\phi$, the charge density is fixed through Eq. (32). Indeed, a charge distribution evolving according to Eq. (38) would not solve the Eqs. (8), (7) (see also Ref. 81 for a similar discussion). Combining (35), 36 we obtain the Einstein relation

$$
D=\frac{\sigma_{\|}(\omega \rightarrow 0,0)}{\chi_{\rho_{\mathcal{Q}} \rho_{\mathcal{Q}}}(0, \mathbf{q} \rightarrow 0)} .
$$

The order of limits for $\omega$ and $\mathbf{q}$ is essential, since at finite $\omega, \mathbf{q}$, Eq. 37 determines the ratio $\sigma_{\|} / \chi_{\rho_{\mathcal{Q}} \rho_{\mathcal{Q}}}$. Finally we note, that even though diffusion is normal for Yukawa interacting charges (see Eq. (30)), the diffusion constant $D_{Y}$ is not equal to the $D$ of Eq. (39).

\section{Gated systems}

Two dimensional solid-state systems can be manipulated by gates [82, 83]. In particular, if the distance between the $2 \mathrm{D}$ channel and the gate is smaller than the length scales of the charge inhomogeneities inside the $2 \mathrm{D}$ layer, the Poisson term (12) on the right of Eq. (8) can be replaced by a capacitive term $\phi_{C}=\rho_{\mathcal{Q}}^{(1)} / C$, where $C$ is the gate capacitance per unit area. This is the local capacitance approximation, which is appropriate for many gated devices $\left[84,87\right.$. The force $-\nabla \phi_{C}$ stemming from the capacitive term can be absorbed into the pressure term of the Navier-Stokes equation with the substitution

$$
K \rightarrow \tilde{K}=K+\frac{\rho_{\mathcal{Q}}^{(0)}}{C} .
$$

The absence of the Poisson term 12 changes the dispersion relations of the hydrodynamic modes. Instead of Eq. 15, the hydrodynamic modes are now determined by

$$
\omega_{g \pm}=-\frac{i+i \nu q^{2}}{2 \tau} \pm \sqrt{\frac{\tilde{K}}{\rho_{\mathcal{M}}^{(0)}} q^{2}-\frac{\left(1+\nu q^{2}\right)^{2}}{4 \tau^{2}}}
$$

The subscript $g$ indicates, that we are considering a gated system with a uniform electrostatic potential. For small $q$ we have

$$
\begin{aligned}
\omega_{g-} & =-\frac{i}{\tau}-i q^{2}\left(\frac{\nu}{\tau}-\frac{\tilde{K} \tau}{\rho_{\mathcal{M}}^{(0)}}\right) \\
\omega_{g+} & =-\frac{i \tilde{K} q^{2} \tau}{\rho_{\mathcal{M}}^{(0)}} .
\end{aligned}
$$

While $\omega_{g-}$ is gapped, $\omega_{g+}$ is an ordinary diffusive mode with a diffusion constant $\tilde{D}=\tilde{K} \tau / \rho_{\mathcal{M}}^{(0)}$. For a gated structure the diffusion constant governing the diffusion of charges is indeed equal to the one obtained from the Einstein relation 39 .

\section{MAGNETIC FIELDS}

Magnetic fields qualitatively change the spectrum of collective excitations of a charged liquid. Under the influence of a magnetic field $B$ charges oscillate at the cyclotron frequency

$$
\omega_{c}=B \rho_{\mathcal{Q}}^{(0)} / \rho_{\mathcal{M}}^{(0)} c
$$

At finite wavevectors, the cyclotron resonance merges with the plasmon and gives rise to the magnetoplasmon mode. We are interested in how the dispersion relations of collectives modes change when both a momentum relaxation time $\tau$ and a uniform magnetic field are added. This can be studied by adding a uniform magnetic field oriented perpendicular to the fluid plane to the NavierStokes equation:

$$
\partial_{t}\left(\rho_{\mathcal{M}} u_{i}\right)+\partial_{j} \Pi_{i j}=-\frac{1}{\tau} \rho_{\mathcal{M}} u_{i}-\rho_{\mathcal{Q}} \nabla \phi+\frac{B}{c} \varepsilon_{i j} j_{\mathcal{Q}, j} .
$$

The last term in Eq. 44 describes the Lorentz force exerted on the fluid by the magnetic field. We assume that the magnetic field is weak, such that Landau quantizuation effects, as well as the localization of electrons on cyclotron orbits can be neglected. Linearizing Eq. 44 
in $u_{i}, \rho_{\mathcal{M}}^{(1)}$ and $\rho_{\mathcal{Q}}^{(1)}$, performing a Fourier transform and writing the equation in terms of matrices, we find

$$
\mathcal{D}(\omega, \mathbf{q}) \mathbf{u}(\omega, \mathbf{q})=0
$$

with

$$
\mathcal{D}=\left[\begin{array}{cc}
\left(-i \omega+\tau^{-1}\right)+\left(\frac{2 a i}{\omega q}+\frac{i K}{\rho_{\mathcal{M}}^{(0)} \omega}+\frac{\zeta}{\rho_{\mathcal{M}}^{(0)}}\right) q_{1}^{2}+\nu q^{2} & \left(\frac{2 a i}{\omega q}+\frac{i K}{\rho_{\mathcal{M}}^{(0)} \omega}+\frac{\zeta}{\rho_{\mathcal{M}}^{(0)}}\right) q_{1} q_{2}-\omega_{c} \\
\left(\frac{2 a i}{\omega q}+\frac{i K}{\rho_{\mathcal{M}}^{(0)} \omega}+\frac{\zeta}{\rho_{\mathcal{M}}^{(0)}}\right) q_{1} q_{2}+\omega_{c} & \left(-i \omega+\tau^{-1}\right)+\left(\frac{2 a i}{\omega q}+\frac{i K}{\rho_{\mathcal{M}}^{(0)} \omega}+\frac{\zeta}{\rho_{\mathcal{M}}^{(0)}}\right) q_{2}^{2}+\frac{\eta}{\rho_{\mathcal{M}}^{(0)}} q^{2}
\end{array}\right]
$$

Here $\nu=\eta / \rho_{\mathcal{M}}^{(0)}$ is the kinematic viscosity. The dispersion relations of collective modes can be found by setting

$$
\operatorname{det}(\mathcal{A})=0
$$

In the limit $\tau \rightarrow \infty$, Eq. 47] gives the magnetoplasmon dispersion [88, 89]

$$
\omega_{m p, \pm}= \pm \sqrt{2 a q+\omega_{c}^{2}}
$$

where the conventional square-root plasmon spectrum is gapped out by the magnetic field. It is interesting to note that in Eq. (48) the two limits $q \rightarrow 0$ and $\omega_{c}^{2} \rightarrow 0$ are not interchangeable, yielding

$$
\omega_{m p,+} \approx \sqrt{2 a q}+\frac{\omega_{c}^{2}}{2 \sqrt{2 a q}}, \quad \omega_{c}^{2} \ll a q
$$

and

$$
\omega_{m p,+} \approx \omega_{c}+a q / \omega_{c}, \quad a q \ll \omega_{c}^{2} .
$$

Either the cyclotron motion or the plasmon waves dominate the collective behavior. This behavior is even more striking at finite $\tau$. The modes $\omega_{-}, \omega_{\perp}$ of the Eqs. (16) and (19) then become

$$
\begin{aligned}
& \omega_{-}^{\mathrm{mag}} \approx-\frac{i}{\tau}-i c_{1}+2 i a \tau\left(1-\omega_{c}^{2} \tau^{2}\right) q-i c_{2} q-i \frac{c_{3}}{q}, \quad \omega_{c}^{2} \ll a q \\
& \omega_{-}^{\mathrm{mag}} \approx-\omega_{c}\left(1+a \tau^{2} q\right)-\frac{i}{\tau}+i a \tau\left(1-\omega_{c}^{2} \tau^{2}\right) q, \quad a q \ll \omega_{c}^{2} \\
& \omega_{\perp}^{\mathrm{mag}} \approx-\frac{i}{\tau}+i c_{1}+i c_{2} q+i \frac{c_{3}}{q}, \quad \omega_{c}^{2} \ll a q \\
& \omega_{\perp}^{\mathrm{mag}} \approx \omega_{c}\left(1+a \tau^{2} q\right)-\frac{i}{\tau}+i a \tau\left(1-\omega_{c}^{2} \tau^{2}\right) q, \quad a q \ll \omega_{c}^{2},
\end{aligned}
$$

where we have used the abbreviations $c_{1}=\omega_{c}^{2} \frac{\zeta-K \tau}{4 a^{2} \tau^{2} \rho_{\mathcal{M}}^{(0)}}, c_{2}=\omega_{c}^{2} \frac{4 a^{2} \tau^{3} \nu \rho_{\mathcal{M}}^{(0)}+\tau^{2} K^{2}+\zeta^{2}-2 \zeta K \tau}{8 a^{3} \tau^{3}\left(\rho_{\mathcal{M}}^{(0)}\right)^{2}}$ and $c_{3}=\frac{\omega_{c}^{2}}{2 a \tau}$. In the limit of small but finite magnetic fields, the modes $\omega_{-}^{\mathrm{mag}}, \omega_{\perp}^{\mathrm{mag}}$ acquire a dispersive real part of $\pm \omega_{c}\left(1+a \tau^{2} q\right)$ and become wavelike, albeit heavily damped. The mode spectrum for larger $q$ is quite complicated and is depicted in Figs. 3 and 4 . The noninterchangeability of the $q \rightarrow 0$ and $\omega_{c}^{2} \rightarrow 0$ limits is a subtle issue since both variables set length scales that influence the transport behavior: either magnetotransport or nonlocal effects dominate. Plasmon spectra for different values of $\omega_{c}$ and $\tau$ are the subject of Ref. [54. The authors point out, that electrodynamic retardation effects may play an important role. For 2D undamped plasmons in the absence of magnetic fields retardation becomes important for $q \rightarrow 0$, when the phase velocity approaches the the speed of light $c$. In the case of damped magnetoplasmons, the interplay between $\omega_{c} \tau, q$ and the dc conductivity $\sigma$ which can approach $c$ becomes important. However, the superdiffusive mode, which is under consideration here, is save from these effects. For the superdiffusive mode (18) the two limits $q \rightarrow 0$ and $\omega_{c}^{2} \rightarrow 0$ are interchageable. In both cases the superdiffusive dispersion reads

$$
\omega_{+}^{\operatorname{mag}}=-2 i a \tau\left(1-\omega_{c}^{2} \tau^{2}\right) q .
$$

The superdiffusion is thus slower by a factor of $1-\omega_{c}^{2} \tau^{2}$ for $\omega_{c} \tau \ll 1$.

\section{LANGEVIN EQUATIONS AND LÉVY FLIGHTS}

Diffusion processes can be modeled with Langevin-type stochastic equations. Here, we demonstrate that charged particles interacting via the Coulomb potential, while un- 

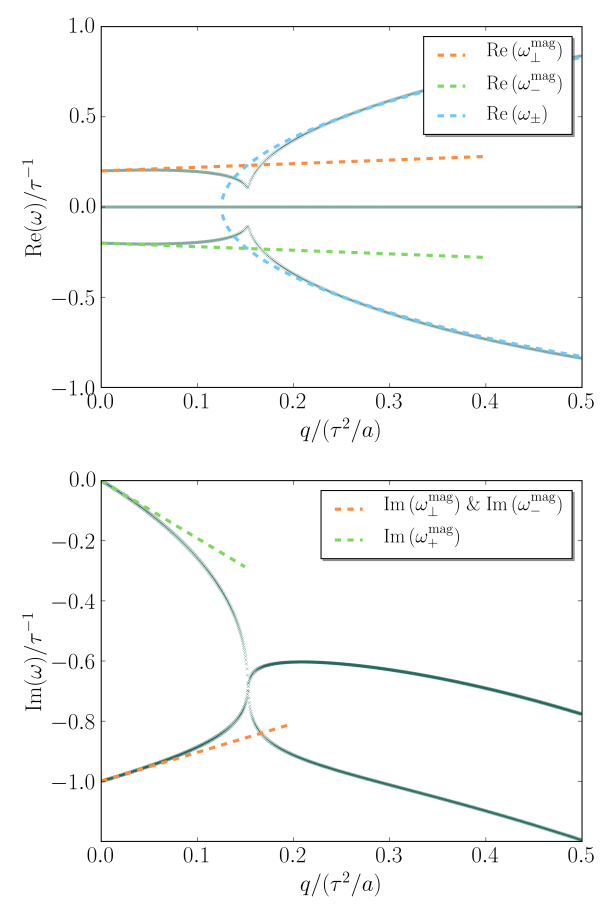

Figure 3. Collective modes of a charged two dimensional liquid in the presence of momentum relaxation and a perpendicular magnetic field. The two damped magnetoplasmon modes $\omega_{\perp}^{\text {mag }}$ and $\omega_{-}^{\text {mag }}$ and the superdiffusive mode $\omega_{+}^{\text {mag }}$ are shown. The colored dashed lines correspond to the approximations of Eqs. (52), (54) and (55). The blue dashed line depicts the damped plasmon dispersion in the absence of magnetic fields $\omega_{ \pm}$given in Eq. 33, which is a reasonable approximation to the magnetoplasmon dispersion at larger $q$. At sufficiently large magnetic fields, the cyclotron resonance and the damped plasmon mode merge. Here $\omega_{c}=0.2 \tau^{-1}$ was chosen, where $\omega_{c}$ is the cyclotron frequency and $\tau^{-1}$ is the rate of momentum relaxation.

dergoing diffusion, indeed follow Lévy flight trajectories that produce the superdiffusive dynamics of Eq. (18). Our starting point are coupled Langevin equations for the particle coordinates $\mathbf{r}_{i}(t)$ [42, 44, 90]:

$$
\ddot{\mathbf{r}}^{(i)}=-\frac{\mathcal{Q}^{2}}{m} \sum_{j \neq i}^{N} \frac{1}{\left|\mathbf{r}^{(j)}-\mathbf{r}^{(i)}\right|}-\frac{1}{\tau} \dot{\mathbf{r}}+\frac{1}{m} \boldsymbol{\eta}^{(i)} .
$$

$\mathcal{Q}$ is the particle charge and $\boldsymbol{\eta}_{i}$ is an uncorrelated stochastic force for which holds $\left\langle\eta_{k}^{(i)}(t) \eta_{l}^{(j)}\left(t^{\prime}\right)\right\rangle=$ $\kappa \delta_{i j} \delta_{k l} \delta\left(t-t^{\prime}\right)$. The Eq. (56) could describe a onecomponent Coulomb plasma [44] (as e.g. realized by macroions in colloidal suspensions [45, 91]). Here $\kappa=$ $2 m k_{B} T \tau^{-1}$ holds due to the Einstein relation.

It is a textbook result that without the Coulomb term in Eq. 56, the particles will undergo Brownian motion. Indeed, for $\mathcal{Q}=0$ the response to the stochastic force is given by

$$
\mathbf{r}^{(i)}(t)=\frac{\tau}{m} \int_{0}^{t} d t^{\prime}\left(1-e^{-\frac{\left(t-t^{\prime}\right)}{\tau}}\right) \boldsymbol{\eta}^{(i)}\left(t^{\prime}\right)
$$
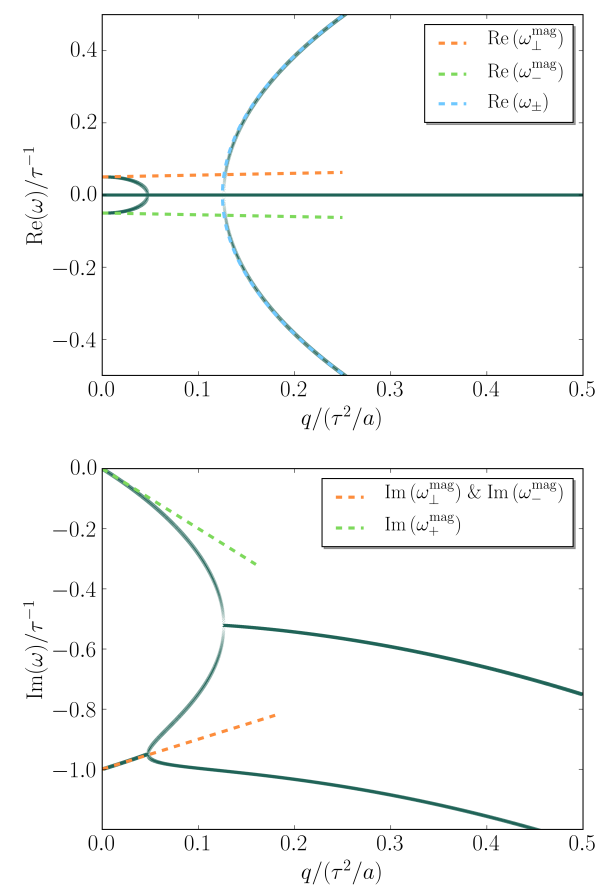

Figure 4. Collective modes of a charged two dimensional liquid in the presence of momentum relaxation and a perpendicular magnetic field. In contrast to Fig. 3, a smaller magnetic field $\left(\omega_{c}=0.05 \tau^{-1}\right)$ was chosen. The two damped magnetoplasmon modes $\omega_{\perp}^{\text {mag }}$ and $\omega_{-}^{\text {mag }}$ and the superdiffusive mode $\omega_{+}^{\mathrm{mag}}$ are shown. The colored dashed lines correspond to the approximations of Eqs. (52), 54) and (55). The blue dashed line depicts the damped plasmon dispersion in the absence of magnetic fields $\omega_{ \pm}$given in Eq. (33), which is a reasonable approximation to the magnetoplasmon dispersion at larger $q$. The cyclotron resonance and the damped plasmon mode are well separated, whereas at larger fields strengths, these modes merge (see Fig. 3).

where we assumed that $\boldsymbol{\eta}$ was switched on at $t=0$. For the variance $\mathbf{r}^{(i)}(t)$ follows

$$
\left\langle\mathbf{r}^{(i)}(t) \cdot \mathbf{r}^{(j)}(t)\right\rangle \approx \delta_{i j} \frac{\tau^{2} \kappa}{m^{2}} t
$$

which corresponds to an ordinary Gaussian diffusion process. To demonstrate, how the non-Gaussian superdiffusive dynamics of Eq. (18) emerges once the Coulomb interactions are turned on, we integrate Eq. (56) numerically. In the simulations, we used periodic boundary conditions and have chosen $t_{c}=\sqrt{m r_{c}^{3} / \mathcal{Q}^{2}}$ as our unit of time. The length $r_{c}$ is chosen arbitrarily (box size $L=25 r_{c}$ ) but can be related to the Wigner-Seitz radius $a: a=\sqrt{\pi n} \approx 1.54 r_{c}$. At $t=0$, the system consists of a uniform background distribution of particles $\rho_{\mathcal{Q}}^{(0)}=0.72 / r_{c}^{2}$ and a small number of non-equlibrium particles $(n=20)$ localized completely within the unit square $\Theta(1-2|x|) \Theta(1-2|y|)$. The coupling parameter $\Gamma$ is small $\Gamma=\mathcal{Q}^{2} /\left(a k_{B} T\right) \approx 0.05$ and the damping is substantial: $\tau=0.1 t_{c}$. 
The movements of the particles at $t>0$ can be interpreted as random walks. From the discussion of Sec. III. one expects that the distances travelled by the particles during an interval $\Delta t$ are distributed according to the Cauchy distribution Eq. (22). I.e., if the diffusion is indeed anomalous with a coefficient $\alpha=1$, the step sizes $\Delta r$ of the random walk will follow a fat-tail powerlaw distribution decaying as

$$
p(\Delta r) \sim \frac{1}{\Delta r^{3}}
$$

The superdiffusive behavior manifests itself at small wavevectors, i.e. large distances, therefore the behavior for small step sizes will deviate from the $\Delta r^{-3}$ law. Since the variance of the Cauchy distribution is not defined, we cannot identify the superdiffusive dynamics by measuring the correlation function $\left\langle\mathbf{r}^{(i)}(t) \cdot \mathbf{r}^{(j)}(t)\right\rangle$. Instead, the step size distribution of Eq. (57) can be used to study the Lévy flight nature of the diffusion process.

Fig. 11 shows the step size distribution obtained in our computational experiment. The power-law decay of Eqs. 22, 57 for large $\Delta r$ is clearly observed. Thus, the diffusive random motion of Coulomb interacting two dimensional particles is a non-Gaussian, Lévy stable random walk. From the general properties of such random walks, we know that the mean travelled distance of a particles grows as $t$, in contrast to the $\sqrt{t}$ scaling of normal diffusion 48, 49.

\section{SPECIFIC HEAT}

Finally, we want to discuss the contributions of the (super)diffusive modes to the specific heat $C$ of a charged two dimensional liquid. The thermodynamics of collective excitation has been the subject of many studies (see e.g. [55, 92, 94]). In Appendix B, we show that the modes' contribution to internal energy density $E$ is given by

$$
E=\int_{0}^{\infty} d \varepsilon \frac{\varepsilon \nu(\varepsilon)}{e^{\beta \varepsilon}-1}
$$

where $\beta=1 / k_{B} T$ and $\nu(\varepsilon)$ is the density of states of the modes:

$$
\nu(\varepsilon)=-\frac{1}{\pi} \int_{0}^{q^{*}} \frac{q d q}{2 \pi} \operatorname{Im}[G(\varepsilon, \mathbf{q})] .
$$

$G(\varepsilon, \mathbf{q})$ is the Green's function of the diffusion equation. $q^{*}$ serves as a momentum cut-off. The specific heat is defined as

$$
c_{V}=\frac{\partial E}{\partial T}
$$

To simplify the analysis, we will focus on low temperatures, where the relevant modes will be the superdiffusive mode of Eq. (18) while the gapped mode (17) will only gain importance at higher temperatures. We begin with the superdiffusive mode $\omega_{+}=-2 i a|q|$ and obtain

$$
\nu(\varepsilon)=\frac{2 a q^{*} \tau-\varepsilon \tan ^{-1}\left(2 a q^{*} \tau / \varepsilon\right)}{8 a^{2} \tau^{2} \pi^{2}} .
$$

The superdiffusive mode contribution to the heat capacity for small temperatures is then given by

$$
c_{V}=c_{1} T-c_{2} T^{2}+c_{3} T^{3}-\mathcal{O}\left(T^{5}\right),
$$

where all higher orders are of odd powers in $T$. For the coefficient of the linear term we obtain $c_{1}=\frac{q^{*}}{24 a \tau}$. Surprisingly, $c_{2}=\frac{3 \zeta(3)}{8 \pi a^{2} \tau^{2}}$ does not depend on the momentum cut-off $q^{*}$ and has a negative sign (although $C$ is always positive). This is due to the fact that the $T^{2}$-dependence can be traced back to the nonanalyticity of the superdiffusive mode at small $q$. We observe that the integrand of Eq. (58) depends on $\varepsilon^{2}$, yet the density of states $\nu(\varepsilon)$ has a linear term in $\varepsilon$. We can extract this term from the integral of Eq. (58):

$$
\begin{aligned}
\lim _{\varepsilon \rightarrow 0} \frac{\nu(\varepsilon)-\nu(0)}{\varepsilon} & =\lim _{\varepsilon \rightarrow 0} \frac{1}{2 \pi a \tau} \int_{0}^{q^{*}} \frac{d q}{2 \pi} \frac{1}{\varepsilon}\left(\frac{4 a^{2} \tau^{2} q^{2}}{\varepsilon^{2}+4 a^{2} \tau^{2} q^{2}}-q\right) \\
& =-\frac{1}{16 \pi a^{2} \tau^{2}} \int_{-\infty}^{\infty} d q \delta(q)
\end{aligned}
$$

For small energies, the DOS is

$$
\nu(\varepsilon) \approx \frac{q^{*}}{4 \pi^{2} a \tau}-\frac{\varepsilon}{16 \pi a^{2} \tau^{2}},
$$

giving the above values of $c_{1}, c_{2}$. It is well known that to leading order at low temperatures, the specific heat $C$ of Galilei invariant Fermi liquids is linear in the temperature, just as for free Fermions. However the analogy does not hold beyond the leading order term. Nonanalytic terms in the fermion self energy of two dimensional Fermi liquids result in corrections to the specific heat $\delta C$ which behave as $\delta C \sim T^{2}$ 95 97. This result is true for both Coulomb and short range interactions [98. Here we show that in the presence of momentum relaxation, the nonanalytic superdiffusive mode of Eq. (18) as well contributes $\mathrm{a} \sim T^{2}$ correction to the specific heat, however with an opposite sign. This is in contrast to the plasmon resonance of a two dimensional charged system where the plasmon dispersion is given by $\omega=\sqrt{2 a q}$ and only contributes a sub-subleading $\sim T^{4}$ term [55].

For the specific heat contribution of the diffusive mode $\omega_{g+}$ of gated $2 \mathrm{D}$ systems we obtain

$$
c_{V, g}=d_{1} T+d_{1}^{\prime} T \log \left(\frac{1}{T}\right)+d_{3} T^{3}+\mathcal{O}\left(T^{5}\right) .
$$

Gating thus qualitatively changes the specific heat of a charged two dimensional system. The low temperature behavior will be dominated by the $T \log (1 / T)$ term. Interestingly, several mechanisms have been discussed that lead to a $T \log (1 / T)$ temperature dependence of the specific heat in two dimensional systems, such as quantum 
critical fluctuations of overdamped bosonic modes with a dynamical exponent $z=2$ [57] and scattering between hot Fermi pocket and cold Fermi surface electrons in $\mathrm{Sr}_{3} \mathrm{Ru}_{2} \mathrm{O}_{7}$ [58]. For $\mathrm{Sr}_{3} \mathrm{Ru}_{2} \mathrm{O}_{7}$, the $T \log (1 / T)$ contribution has been observed experimentally [56].

\section{ACKNOWEDGEMENTS}

I am greatful to Michael Bonitz, Igor Gornyi and Jörg Schmalian for helpful comments and discussions. I also want thank Bhilahari Jeevanesan and Jonas Karcher who helped to improve this manuscript.

\section{Appendix A: Charge and mass densities in systems without Galilean invariance}

In Galilean invariant systems the notions of mass and charge densities are straightforward. If $\rho$ is the particle number density, the mass density is given by $\rho_{\mathcal{M}}=m \rho$ and the charge density by $\rho_{\mathcal{Q}}=e \rho$, where $m$ and $e$ are the mass and charge of a particle. However many solid state systems do not exhibit Galilean invariance, and it is usefull to extend the definitions of mass and charge densities to non-Galilean invariant, yet translation invariant systems, where momentum conservation ensures the validity of hydrodynamics. Here the velocity $u_{i}(\mathbf{x})$ is defined as a source of the conserved crystal momentum [38. With the shift

$$
H \rightarrow H-\int d^{2} x u_{i}(t, \mathbf{x}) P_{i}(\mathbf{x}),
$$

where $H$ is the full Hamiltonian of the system and $P_{i}(\mathbf{x})$ is the momentum operator, the densities $\rho_{\mathcal{M}}(t, \mathbf{x})$, $\rho_{\mathcal{Q}}(t, \mathbf{x})$ can be defined as response functions and calculated using the memory matrix formalism [21, 38]. Memory matrices allow to construct a hydrodynamic approximation to a quantum system by restricting the infinitedimensional space of possible observables to a few conserved quantities and quantities which decay at very long time scales. Sticking to the notation of Ref. [38, we will call these quantities $X_{A}$. Their thermodynamic conjugate shall be called $U_{B}$. An important object is the generalized conductivity $\sigma_{A B}$. The memory matrix formalism provides efficient means for its calculation. The generalized conductivity relates the quantities $X_{A}$ to the fields $\dot{U}_{B}$ :

$$
\left\langle X_{A}\right\rangle=-\sigma_{A B} \dot{U}_{B}
$$

where a summation over the index $B$ labelling the (quasi)conserved quantities is implied. $\sigma_{A B}$ can be expressed in terms of retarded Green's functions

$$
\sigma_{A B}(z, \mathbf{q})=\frac{1}{i z}\left(G_{A B}^{R}(z, \mathbf{q})-G_{A B}^{R}(i 0, \mathbf{q})\right)
$$

with

$$
G_{A B}^{R}(t, \mathbf{x})=-i \Theta(t)\left\langle\left[X_{A}(t, \mathbf{x}), X_{B}(0, \mathbf{0})\right]\right\rangle .
$$

As is customary in memory matrix literature, we used the Laplace transform

$$
G_{A B}^{R}(z, \mathbf{q})=\int_{0}^{\infty} d t e^{i z t} G_{A B}^{R}(t, \mathbf{q}) .
$$

In our case the quantities $X_{A}$ include the momentum $P_{i}$, which, following Eq. A1, is sourced by the velocity $u_{i}$. Using Eq. A2, we write

$$
\left\langle P_{i}\right\rangle=-i z \sigma_{P_{i}, u_{i}} u_{i} .
$$

The above equation suggests that the mass density should be defined as $\rho_{\mathcal{M}}=-i z \sigma_{P_{i}, u_{i}}$. It follows

$$
\left\langle P_{i}\right\rangle(t, \mathbf{x})=\int d t^{\prime} d^{2} x^{\prime} \rho_{\mathcal{M}}\left(t-t^{\prime}, \mathbf{x}-\mathbf{x}^{\prime}\right) u_{i}\left(t^{\prime}, \mathbf{x}^{\prime}\right) .
$$

Finally, we should keep in mind that the scales of hydrodynamic temporal and spatial inhomogeneities $t_{\mathrm{h}}$ and $l_{\mathrm{h}}$ are much smaller than any time or length scale $\tilde{t}, \tilde{l}$ characterizing the Hamiltonian $H$. Since the Green's functions in the Eq. (A3) are calculated at vanishing flow velocities, they will decay on scales given by $\left|t-t^{\prime}\right| \approx \tilde{t}$, $\left|\mathbf{x}-\mathbf{x}^{\prime}\right| \approx \tilde{l}$. On the other hand, the flow velocity $u_{i}$ varies on scales $t_{\mathrm{h}}, l_{\mathrm{h}}$. Thus, in the hydrodynamic limit $\tilde{t} \ll t_{h}, \tilde{l} \ll l_{h}$, Eq. A5 can be approximated by the local relation

$$
g_{i}(t, \mathbf{x}) \approx \rho_{\mathcal{M}}(t, \mathbf{x}) u_{i}(t, \mathbf{x})
$$

$\rho_{\mathcal{M}}(t, \mathbf{x})$ is the mass density used throughout the text. Similarely, we arrive at $\rho_{\mathcal{Q}}=-i z \sigma_{J_{\mathcal{Q}, i}, u_{i}}$, where $J_{\mathcal{Q}, i}$ is the electric current operator, and finally

$$
j_{\mathcal{Q}, i} \approx \rho_{\mathcal{Q}}(t, \mathbf{x}) u_{i}(t, \mathbf{x}) .
$$

\section{Appendix B: Specific heat contribution of collective modes}

We begin with the partition function

$$
Z=\int \mathcal{D}\left[\phi_{\mathbf{q}}\right] e^{-\sum_{\mathbf{q}, n} \phi_{\mathbf{q}, i \omega_{n}} G^{-1}\left(i \omega_{n}, \mathbf{q}\right) \phi_{\mathbf{q}, i \omega_{n}}}
$$

Here, $G\left(i \omega_{n}, \mathbf{q}\right)$ is the Green's function of the damped bosonic plasmon mode

$$
G\left(i \omega_{n}, \mathbf{q}\right)=\frac{1}{-i \omega_{n}+\omega_{+}(\mathbf{q})},
$$

where $\omega_{+}(\mathbf{q})$ was introduced in Eq. (18). $\phi_{\mathbf{q}}$ is representing the bosonic plasmon fields. The heat capacity can be calculated from the internal energy $E$, which is given by 99 , 100]

$$
E=-\frac{\partial}{\partial \beta} \int \frac{d^{2} q}{(2 \pi)^{2}} \sum_{n} \ln \left[\beta G^{-1}\left(i \omega_{n}, \mathbf{q}\right)\right] .
$$


First, we evaluate the Matsubara sum over bosonic frequencies $\omega_{n}=2 \pi n / \beta$ by rewriting it as a contour integral around the imaginary axis of a variable $\varepsilon$ :

$E=-\frac{\partial}{\partial \beta} \int \frac{d^{2} q}{(2 \pi)^{2}} \int_{\mathcal{C}} \frac{d \varepsilon}{(2 \pi i)} \frac{\beta}{e^{\beta \varepsilon}-1} \ln \left[\beta\left(-\varepsilon+\omega_{+}(\mathbf{q})\right)\right]$

In the following, it will be convenient to use the abbreviation

$$
\omega_{+}(\mathbf{q}) \approx-2 i a \tau_{1} q \equiv \xi
$$

The integrand has a branch cut at $\operatorname{Re} \varepsilon>0$ and $\operatorname{Im} \varepsilon=\xi$. Correspondingly, the contour can be deformed such that it encircles the line $\operatorname{Im} \varepsilon=-2 i a \tau_{1} q$ running from 0 to infinity and back. The internal energy is then given by

$$
E=-\frac{\partial}{\partial \beta} \int \frac{d^{2} q}{(2 \pi)^{2}} \int_{0}^{\infty} \frac{d \varepsilon}{(2 \pi i)}\left\{\frac{\beta}{e^{\beta(\varepsilon+\xi)}-1} \ln \left[\beta\left(-\left(\varepsilon+\xi+i 0^{+}\right)+\xi\right)\right]-\frac{\beta}{e^{\beta(\varepsilon+\xi)}-1} \ln \left[\beta\left(-\left(\varepsilon+\xi-i 0^{+}\right)+\xi\right)\right]\right\}
$$

or

$$
E=-\frac{\partial}{\partial \beta} \int \frac{d^{2} q}{(2 \pi)^{2}} \int_{0}^{\infty} \frac{d \varepsilon}{\pi} \frac{\beta}{e^{\beta \varepsilon}-1} \operatorname{Im}(\ln [\beta(-\varepsilon+\xi)])
$$

Simplifying the expression we obtain

$$
\begin{aligned}
E=-\frac{\partial}{\partial \beta} \int \frac{d^{2} q}{(2 \pi)^{2}} \int_{0}^{\infty} \frac{d \varepsilon}{\pi}\left\{\frac{\partial}{\partial \varepsilon}\left(\ln \left(1-e^{\beta \varepsilon}\right)-\beta \varepsilon\right)\right. \\
\quad \times \operatorname{Im}(\ln [\beta(-\varepsilon+\xi)])\} \\
=\frac{\partial}{\partial \beta} \int \frac{d^{2} q}{(2 \pi)^{2}} \int_{0}^{\infty} \frac{d \varepsilon}{\pi}\left\{\left(\ln \left(1-e^{\beta \varepsilon}\right)-\beta \varepsilon\right)\right. \\
\left.\quad \times \operatorname{Im}\left(\frac{1}{-\varepsilon+\xi}\right)\right\} \\
=-\int \frac{d^{2} q}{(2 \pi)^{2}} \int_{0}^{\infty} \frac{d \varepsilon}{\pi} \frac{\varepsilon}{e^{\beta \varepsilon}-1} \operatorname{Im} G(\varepsilon, \mathbf{q})
\end{aligned}
$$

Keeping in mind that the imaginary part of the Green's function determines the spectral function $A(\varepsilon, \mathbf{q})$ via

$$
-\frac{1}{\pi} \operatorname{Im} G(\varepsilon, \mathbf{q})=A(\varepsilon, \mathbf{q}),
$$

and the density of states $\nu(\varepsilon)$ is given by

$$
\nu(\varepsilon)=\int \frac{d^{2} q}{(2 \pi)^{2}} A(\varepsilon, \mathbf{q}),
$$

formula (B7) can be interpreted as an energy average over the Bose-Einstein distribution weightened by the density of states:

$$
E=\int_{0}^{\infty} d \varepsilon \frac{\varepsilon \nu(\varepsilon)}{e^{\beta \varepsilon}-1} .
$$

[1] L. Wang, I. Meric, P. Y. Huang, Q. Gao, Y. Gao, H. Tran, T. Taniguchi, K. Watanabe, L. M. Campos, D. A. Muller, J. Guo, P. Kim, J. Hone, K. L. Shepard, and C. R. Dean, One-dimensional electrical contact to a two-dimensional material, Science 342, 614 (2013).

[2] M. J. M. de Jong and L. W. Molenkamp, Hydrodynamic electron flow in high-mobility wires, Phys. Rev. B 51, 13389 (1995).

[3] J. A. Sulpizio, L. Ella, A. Rozen, J. Birkbeck, D. J. Perello, D. Dutta, M. Ben-Shalom, T. Taniguchi, K. Watanabe, T. Holder, R. Queiroz, A. Principi, A. Stern, T. Scaffidi, A. K. Geim, and S. Ilani, Visualizing poiseuille flow of hydrodynamic electrons, Nature 576, 75 (2019).

[4] G. Gusev, A. Jaroshevich, A. Levin, Z. Kvon, and A. Bakarov, Stokes flow around an obstacle in viscous two-dimensional electron liquid, Scientific Reports 10, 1 (2020).

[5] D. A. Bandurin, I. Torre, R. K. Kumar, M. B. Shalom, A. Tomadin, A. Principi, G. H. Auton, E. Khestanova,
K. S. Novoselov, I. V. Grigorieva, L. A. Ponomarenko, A. K. Geim, and M. Polini, Negative local resistance caused by viscous electron backflow in graphene, Science 351, 1055 (2016).

[6] R. K. Kumar, D. A. Bandurin, F. M. D. Pellegrino, Y. Cao, A. Principi, H. Guo, G. H. Auton, M. B. Shalom, L. A. Ponomarenko, G. Falkovich, K. Watanabe, T. Taniguchi, I. V. Grigorieva, L. S. Levitov, M. Polini, and A. K. Geim, Superballistic flow of viscous electron fluid through graphene constrictions, Nature Physics 13, 1182 (2017).

[7] R. N. Gurzhi, Minimum of resistance in impurity-free conductors, Zh. Eksp. Teor. Fiz. 44, 771 (1963).

[8] R. Gurhzi, Hydrodynamic effects in solids at low temperature, Sov. Phys. Usp. 11, 255 (1968).

[9] R. Bistritzer and A. H. MacDonald, Moiré bands in twisted double-layer graphene, Proceedings of the National Academy of Sciences 108, 12233 (2011).

[10] Y. Cao, V. Fatemi, S. Fang, K. Watanabe, T. Taniguchi, E. Kaxiras, and P. Jarillo-Herrero, Unconventional su- 
perconductivity in magic-angle graphene superlattices, Nature 556, 43 (2018).

[11] Y. Cao, D. Chowdhury, D. Rodan-Legrain, O. RubiesBigorda, K. Watanabe, T. Taniguchi, T. Senthil, and P. Jarillo-Herrero, Strange metal in magic-angle graphene with near planckian dissipation, Physical Review Letters 124, 076801 (2020).

[12] A. P. Mackenzie, The properties of ultrapure delafossite metals, Rep. Prog. Phys. 80, 32501 (2017).

[13] P. J. W. Moll, P. Kushwaha, N. Nandi, B. Schmidt, and A. P. Mackenzie, Evidence for hydrodynamic electron flow in $\mathrm{PdCoO}_{2}$, Science 351, 1061 (2016).

[14] N. Nandi, T. Scaffidi, P. Kushwaha, S. Khim, M. E. Barber, V. Sunko, F. Mazzola, P. D. King, H. Rosner, P. J. Moll, M. König, J. E. Moore, S. Hartnoll, and A. P. Mackenzie, Unconventional magneto-transport in ultrapure $\mathrm{PdCoO}_{2}$ and $\mathrm{PtCo} \mathrm{O}_{2}$, npj Quantum Materials 3, 1 (2018).

[15] N. Nandi, T. Scaffidi, S. Khim, P. Kushwaha, J. Moore, and A. MacKenzie, Size restricted magnetotransport in the non-magnetic delafossite metals $\mathrm{PdCoO}_{2}$ and $\mathrm{PtCoO}_{2}$, APS 2019, P40 (2019).

[16] A. V. Andreev, S. A. Kivelson, and B. Spivak, Hydrodynamic description of transport in strongly correlated electron systems, Phys. Rev. Lett. 106, 256804 (2011).

[17] U. Briskot, M. Schütt, I. V. Gornyi, M. Titov, B. N. Narozhny, and A. D. Mirlin, Collision-dominated nonlinear hydrodynamics in graphene, Phys. Rev. B 92, 115426 (2015).

[18] R. D'Agosta and M. Di Ventra, Hydrodynamic approach to transport and turbulence in nanoscale conductors, Journal of Physics: Condensed Matter 18, 11059 (2006).

[19] L. V. Delacrétaz, B. Goutéraux, S. A. Hartnoll, and A. Karlsson, Theory of hydrodynamic transport in fluctuating electronic charge density wave states, Physical Review B 96, 195128 (2017).

[20] A. Eguiluz and J. Quinn, Hydrodynamic model for surface plasmons in metals and degenerate semiconductors, Physical Review B 14, 1347 (1976).

[21] D. Forster, Hydrodynamic Fluctuations, Broken Symmetry, and Correlation Functions, CRC Press (2018). ISBN 978-0367091323.

[22] V. Galitski, M. Kargarian, and S. Syzranov, Dynamo effect and turbulence in hydrodynamic weyl metals, Physical review letters 121, 176603 (2018).

[23] S. Grozdanov, A. Lucas, and N. Poovuttikul, Holography and hydrodynamics with weakly broken symmetries, Physical Review D 99, 086012 (2019).

[24] T. Holder, R. Queiroz, T. Scaffidi, N. Silberstein, A. Rozen, J. A. Sulpizio, L. Ella, S. Ilani, and A. Stern, Ballistic and hydrodynamic magnetotransport in narrow channels, Phys. Rev. B 100, 245305 (2019).

[25] V. O. A. Hui, S. Lederer and E.-A. Kim, Quantum aspects of hydrodynamic transport from weak electronimpurity scattering, Phys. Rev. B 101, 121107 (2020).

[26] J. M. Link, B. N. Narozhny, E. I. Kiselev, and J. Schmalian, Out-of-bounds hydrodynamics in anisotropic dirac fluids, Phys. Rev. Lett. 120, 196801 (2018).

[27] A. Lucas and K. C. Fong, Hydrodynamics of electrons in graphene, Journal of Physics: Condensed Matter 30, 53001 (2018).

[28] A. Lucas and S. D. Sarma, Electronic sound modes and plasmons in hydrodynamic two-dimensional metals,
Phys. Rev. B 97, 115449 (2018).

[29] A. Lucas and S. A. Hartnoll, Resistivity bound for hydrodynamic bad metals, PNAS 43, 11344 (2017).

[30] R. Moessner, P. Surówka, and P.Witkowski, Pulsating flow and boundary layers in viscous electronic hydrodynamics, Phys. Rev. B 97, 161112 (2018).

[31] B. N. Narozhny, I. V. Gornyi, and A. D. Mirlin, Hydrodynamic approach to electronic transport in graphene, Ann. d. Phys. 529, 1700043 (2017).

[32] A. Principi and G. Vignale, Violation of the wiedemannfranz law in hydrodynamic electron liquids, Phys. Rev. Lett. 115, 056603 (2015).

[33] T. Scaffidi, N. Nandi, B. Schmidt, A. P. Mackenzie, and J. E. Moore, Hydrodynamic electron flow and hall viscosity, Phys. Rev. Lett. 118, 226601 (2017).

[34] D. Svintsov, Hydrodynamic-to-ballistic crossover in dirac materials, Phys. Rev. B 97, 121405 (2018).

[35] D. Svintsov, Emission of plasmons by drifting dirac electrons: A hallmark of hydrodynamic transport, Phys. Rev. B 100, 195428 (2019).

[36] G. Varnavides, A. S. Jermyn, P. Anikeeva, C. Felser, and P. Narang, Electron hydrodynamics in anisotropic materials, Nature Communications 11, 1 (2020).

[37] T. Zdyrski and J. McGreevy, Effects of dissipation on solitons in the hydrodynamic regime of graphene, Phys. Rev. B 99, 235435 (2019).

[38] A. Lucas and S. Sachdev, Memory matrix theory of magnetotransport in strange metals, Physical Review B 91, $195122(2015)$.

[39] A. Buchel and M. Baggioli, Holographic viscoelastic hydrodynamics, Journal of High Energy Physics 2019, 146 (2019).

[40] M. D'yakonov and A. Furman, Charge relaxation in an anisotropic medium and in low-dimensional media, $\mathrm{Zh}$. Eksp. Teor. Fiz 92, 1012 (1987).

[41] B. Liu and J. Goree, Superdiffusion in two-dimensional yukawa liquids, Physical Review E 75, 016405 (2007).

[42] T. Ott and M. Bonitz, Is diffusion anomalous in twodimensional yukawa liquids?, Physical review letters 103, 195001 (2009).

[43] See this Ref. for a review of literature on anomalous diffusion in dusty plasmas.

[44] T. Ott, M. Bonitz, L. Stanton, and M. Murillo, Coupling strength in coulomb and yukawa one-component plasmas, Physics of Plasmas 21, 113704 (2014).

[45] P. B. Warren, A theory of void formation in chargestabilized colloidal suspensions at low ionic strength, The Journal of Chemical Physics 112, 4683 (2000).

[46] M. Kwaśnicki, Ten equivalent definitions of the fractional Laplace operator, Fractional Calculus and Applied Analysis 20, 7 (2017).

[47] S. G. Samko, A. A. Kilbas, and O. I. Marichev, Fractional integrals and derivatives, Taylor \& Francis (1993). ISBN: 978-2881248641.

[48] J.-P. Bouchaud and A. Georges, Anomalous diffusion in disordered media: Statistical mechanisms, models and physical applications, Physics Reports 195, 127 (1990).

[49] B. V. Gnedenko and A. N. Kolmogorov, Limit Distributions for Sums of Independent Random Variables, Adison Wesley (1954).

[50] R. Metzler, E. Barkai, and J. Klafter, Anomalous diffusion and relaxation close to thermal equilibrium: A fractional fokker-planck equation approach, Phys. Rev. Lett. 82, 3563 (1999). 
[51] R. Metzler and J. Klafter, The random walk's guide to anomalous diffusion: a fractional dynamics approach, Phys. Rep. 339, 1 (2000).

[52] A. Lucas, Sound waves and resonances in electron-hole plasma, Physical Review B 93, 245153 (2016).

[53] E. B. Kolomeisky and J. P. Straley, Relaxation of charge in monolayer graphene: Fast nonlinear diffusion versus coulomb effects, Physical Review B 95, 045415 (2017).

[54] V. Volkov and A. Zabolotnykh, Undamped relativistic magnetoplasmons in lossy two-dimensional electron systems, Physical Review B 94, 165408 (2016).

[55] R. A. Höpfel, E. Vass, and E. Gornik, Thermal excitation of two-dimensional plasma oscillations, Physical Review Letters 49, 1667 (1982).

[56] D. Sun, A. Rost, R. Perry, A. Mackenzie, and M. Brando, Low temperature thermodynamic investigation of the phase diagram of $\mathrm{Sr}_{3} \mathrm{Ru}_{2} \mathrm{O}_{7}$, Physical Review B 97, 115101 (2018).

[57] A. Millis, Effect of a nonzero temperature on quantum critical points in itinerant fermion systems, Physical Review B 48, 7183 (1993).

[58] C. H. Mousatov, E. Berg, and S. A. Hartnoll, Theory of the strange metal $\mathrm{Sr}_{3} \mathrm{Ru}_{2} \mathrm{O}_{7}$, Proceedings of the National Academy of Sciences 117, 2852 (2020).

[59] M. Mittendorff, T. Winzer, E. Malic, A. Knorr, C. Berger, W. A. de Heer, H. Schneider, M. Helm, and S. Winnerl, Anisotropy of excitation and relaxation of photogenerated charge carriers in graphene, Nano letters 14, 1504 (2014).

[60] P. Houston and A. Evans, Electron drift velocity in $n$ gaas at high electric fields, Solid-State Electronics 20, 197 (1977).

[61] R. Höpfel, J. Shah, D. Block, and A. Gossard, Picosecond time-of-flight measurements of minority electrons in gaas/algaas quantum well structures, Applied physics letters 48, 148 (1986).

[62] L. D. Landau and E. M. Lifshits, Fluid mechanics, Butterworth-Heinemann (1987). ISBN 978-0750627672.

[63] G. Batchelor, An introduction to fluid dynamics, Cambridge University Press (2000). ISBN 978-0521663960.

[64] V. Silin, Theory of a degenerate electron liquid, JETP 6, 387 (1958).

[65] A. A. Vlasov, On vibration properties of electron gas, J. Exp. Theor. Phys. 8, 291 (1938).

[66] F. Stern, Polarizability of a two-dimensional electron gas, Physical Review Letters 18, 546 (1967).

[67] G. Zala, B. Narozhny, and I. Aleiner, Interaction corrections at intermediate temperatures: Longitudinal conductivity and kinetic equation, Physical Review B 64, 214204 (2001).

[68] M. Giona and H. E. Roman, Fractional diffusion equation for transport phenomena in random media, Physica A: Statistical Mechanics and its Applications 185, 87 (1992).

[69] E. Scalas, R. Gorenflo, and F. Mainardi, Fractional calculus and continuous-time finance, Physica A: Statistical Mechanics and its Applications 284, 376 (2000).

[70] M. Baggioli, G. La Nave, and P. W. Phillips, Fractional hydrodynamics and anomalous diffusion, arXiv preprint arXiv:2006.10064 (2020).

[71] J. Desbois, Algebraic areas distributions for twodimensional Levy flights, Journal of Physics A: Mathematical and General 25, L755 (1992).
[72] E. I. Kiselev and J. Schmalian, Nonlocal hydrodynamic transport and collective excitations in dirac fluids, Physical Review B 102, 245434 (2020).

[73] L. Fritz, J. Schmalian, M. Müller, and S. Sachdev, Quantum critical transport in clean graphene, Phys. Rev. B 78, 85416 (2008).

[74] E. I. Kiselev and J. Schmalian, Lévy flights and hydrodynamic superdiffusion on the dirac cone of graphene, Phys. Rev. Lett. 123, 195302 (2019).

[75] J. Pizarro, M. Rösner, R. Thomale, R. Valentí, and $\mathrm{T}$. Wehling, Internal screening and dielectric engineering in magic-angle twisted bilayer graphene, Physical Review B 100, 161102(R) (2019).

[76] Z. A. Goodwin, F. Corsetti, A. A. Mostofi, and J. Lischner, Attractive electron-electron interactions from internal screening in magic-angle twisted bilayer graphene, Physical Review B 100, 235424 (2019).

[77] S. Hamaguchi and R. Farouki, Thermodynamics of strongly-coupled yukawa systems near the onecomponent-plasma limit. $i$. derivation of the excess energy, The Journal of chemical physics 101, 9876 (1994).

[78] Y. Feng, J. Goree, and B. Liu, Longitudinal viscosity of two-dimensional yukawa liquids, Physical Review E 87, 013106 (2013).

[79] G. Kalman, P. Hartmann, Z. Donkó, and M. Rosenberg, Two-dimensional yukawa liquids: Correlation and dynamics, Physical review letters 92, 065001 (2004).

[80] D. Pines and P. Nozières, Theory Of Quantum Liquids: Normal Fermi Liquids, CRC Press (1989). ISBN 9780201407747.

[81] A. Efros, Negative density of states: Screening, einstein relation, and negative diffusion, Physical Review B 78, 155130 (2008).

[82] M. S. Shur, GaAs devices and circuits, Springer Science \& Business Media (2013).

[83] P. V. Nguyen, N. C. Teutsch, N. P. Wilson, J. Kahn, X. Xia, A. J. Graham, V. Kandyba, A. Giampietri, A. Barinov, G. C. Constantinescu et al., Visualizing electrostatic gating effects in two-dimensional heterostructures, Nature 572, 220 (2019).

[84] M. Dyakonov and M. Shur, Shallow water analogy for a ballistic field effect transistor: New mechanism of plasma wave generation by dc current, Physical review letters 71, 2465 (1993).

[85] M. Dyakonov and M. Shur, Detection, mixing, and frequency multiplication of terahertz radiation by twodimensional electronic fluid, IEEE transactions on electron devices 43, 380 (1996).

[86] I. Torre, L. V. de Castro, B. V. Duppen, D. B. Ruiz, F. M. Peeters, F. H. L. Koppens, and M. Polini, Acoustic plasmons at the crossover between the collisionless and hydrodynamic regimes in two-dimensional electron liquids, Phys. Rev. B 99, 144307 (2019).

[87] A. Zabolotnykh and V. Volkov, Interaction of gated and ungated plasmons in two-dimensional electron systems, Physical Review B 99, 165304 (2019).

[88] N. J. M. Horing and M. M. Yildiz, Quantum theory of longitudinal dielectric response properties of a two-dimensional plasma in a magnetic field, Annals of Physics 97, 216 (1976).

[89] D. Mast, A. Dahm, and A. Fetter, Observation of bulk and edge magnetoplasmons in a two-dimensional electron fluid, Physical review letters 54, 1706 (1985). 
[90] S. Chandrasekhar, Stochastic problems in physics and astronomy, Rev. Mod. Phys. 15, 1 (1943).

[91] E. C. Oğuz, R. Messina, and H. Löwen, Multilayered crystals of macroions under slit confinement, Journal of Physics: Condensed Matter 21, 424110 (2009).

[92] K. Trachenko and V. Brazhkin, Collective modes and thermodynamics of the liquid state, Reports on Progress in Physics 79, 016502 (2015).

[93] M. Baggioli and A. Zaccone, Universal origin of boson peak vibrational anomalies in ordered crystals and in amorphous materials, Physical review letters $\mathbf{1 2 2}$, 145501 (2019).

[94] M. Baggioli and A. Zaccone, Hydrodynamics of disordered marginally stable matter, Physical Review Research 1, 012010 (2019).
[95] D. Belitz, T. R. Kirkpatrick, and T. Vojta, Nonanalytic behavior of the spin susceptibility in clean fermi systems, Physical Review B 55, 9452 (1997).

[96] A. V. Chubukov and D. L. Maslov, Nonanalytic corrections to the fermi-liquid behavior, Physical Review $\mathrm{B}$ 68, 155113 (2003).

[97] A. V. Chubukov and D. L. Maslov, Singular corrections to the fermi-liquid theory, Physical Review B 69, 121102 (2004).

[98] A. V. Chubukov, D. L. Maslov, S. Gangadharaiah, and L. I. Glazman, Singular perturbation theory for interacting fermions in two dimensions, Physical Review B 71, 205112 (2005).

[99] A. Altland and B. D. Simons, Condensed matter field theory, Cambridge university press (2010). ISBN: 9780521769754.

[100] J. W. Negele and H. Orland, Quantum many-particle systems, CRC Press (2018). 